\title{
Generation of dissipative solitons in normal- dispersion Raman fiber laser
}

\author{
U. Tegin ${ }^{1}$, P. Elahi ${ }^{1}$, C. Senel ${ }^{2}$, E. Ergecen ${ }^{3}$ and F. Ömer Ilday ${ }^{1,4}$ \\ ${ }^{1}$ Department of Physics, Bilkent University, 06800 Ankara, Turkey \\ ${ }^{2}$ TUBITAK National Metrology Institute (UME), PO 54, TR-41470, Gebze, Kocaeli, Turkey \\ ${ }^{3}$ Department of Electrical and Electronics Engineering, METU Ankara, 06800, Turkey \\ ${ }^{4}$ Department of Electrical and Electronics Engineering, Bilkent University, 06800 Ankara, Turkey
}

\begin{abstract}
Dissipative soliton pulses in a synchronously pumped all-normal-dispersion Raman fiber laser is presented theoretically and experimentally. The laser generates $7.1 \mathrm{~nJ}$ intra-cavity pulses at $1.12 \mu \mathrm{m}$ and is compressed to $136 \mathrm{fs}$.

Stolen, et al. introduced synchronously pumped oscillators based on stimulated Raman scattering (SRS) in 1977 [1]. Raman lasers generate wavelengths outside of the limited range covered by the conventional laser gain media. Generally, Raman lasers are pumped by another laser source to generate a frequency-shifted Stokes (or anti Stokes) wave. Raman solitons, which are generated by this technique, require $\mathrm{km}$-long cavities possess anomalous-dispersion fibers and usually are limited to generate high energy pulses [2]. Self-similar pulse evolution has been generated in $\mathrm{km}-$ long fibers to achieve picosecond-long pulses [3].

Here, we present a new type of Raman oscillator, which supports dissipative solitons in all-normaldispersion regime. The dynamic of the system is governed by the complex Ginzburg-Landau equation which can support dissipative soliton.

The experimental setup shown in Fig. 1 and comprises of a pump laser source to generate highly positively chirped pulses centered at $1065 \mathrm{~nm}$, an amplifier and Raman oscillator. When the Stokes pulse, generated in Raman oscillator, is band-pass filtered and re-launch into the oscillator, the strong Raman pulses are generated.
\end{abstract}

In this experiment, the pump laser is a modelocked oscillator, which generates 6.5 ps long pulses with $37 \mathrm{MHz}$ repetition rate at $1065 \mathrm{~nm}$. The output from oscillator then amplified to $215 \mathrm{~mW}$.

The chirped pulses from amplifier are led into the all-polarization-maintaining Yb-doped fiber Raman oscillator and amplified to $760 \mathrm{~mW}$ to generate the first Stokes wave. The Stokes component is fed back into the Raman oscillator while the pump is filtering out. We employed a precise translation stage to synchronize pump and Stokes and confirmed it by time-domain measurement.

When temporal pump-Raman overlap is achieved, the amplified spectrum is modified dramatically and most of the energy being transferred to the Stokes wave at $1120 \mathrm{~nm}$ (Fig. 2.b). By using of diffraction gratings, the pulses are compressed to 136 fs (Fig. 2.c) which is the shortest achieved dissipative soliton Raman pulse to the best of our knowledge.

Numerical simulation is employed to investigate the pulse dynamic through the system starting from modelocked oscillator, amplifier and Raman oscillator. The output from each stage serves as the initial condition for the next one. The pulse propagation is modeled by generalized nonlinear Schrödinger equation (GNLSE) including gain saturation and Raman scattering and solved by using fourth-order Runge-Kutta in the interaction picture method [5].

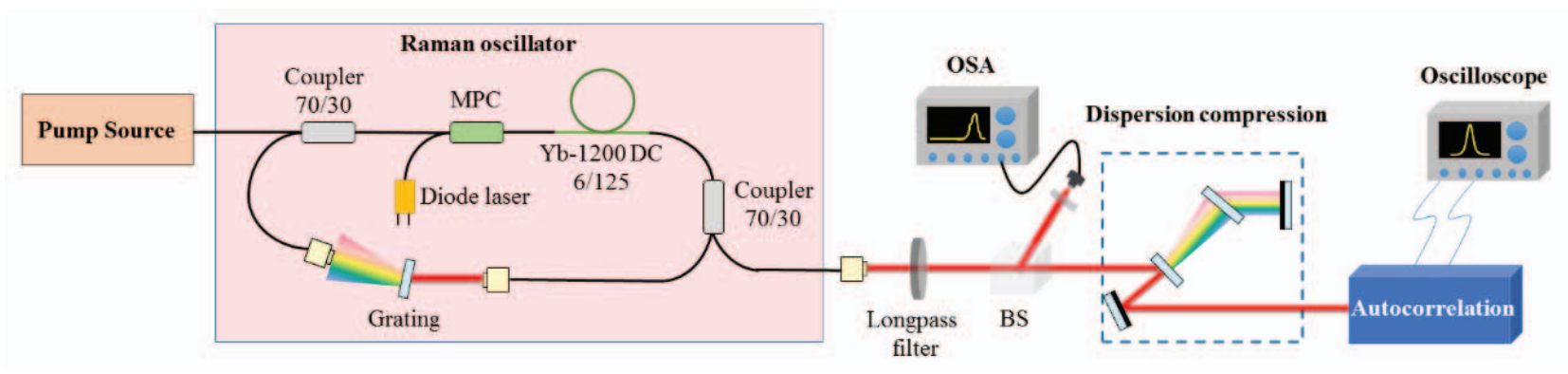

Fig. 1. Schematic experimental setup. MPC, multi-pump signal combiner; DC, double clad; BS, beam splitter 

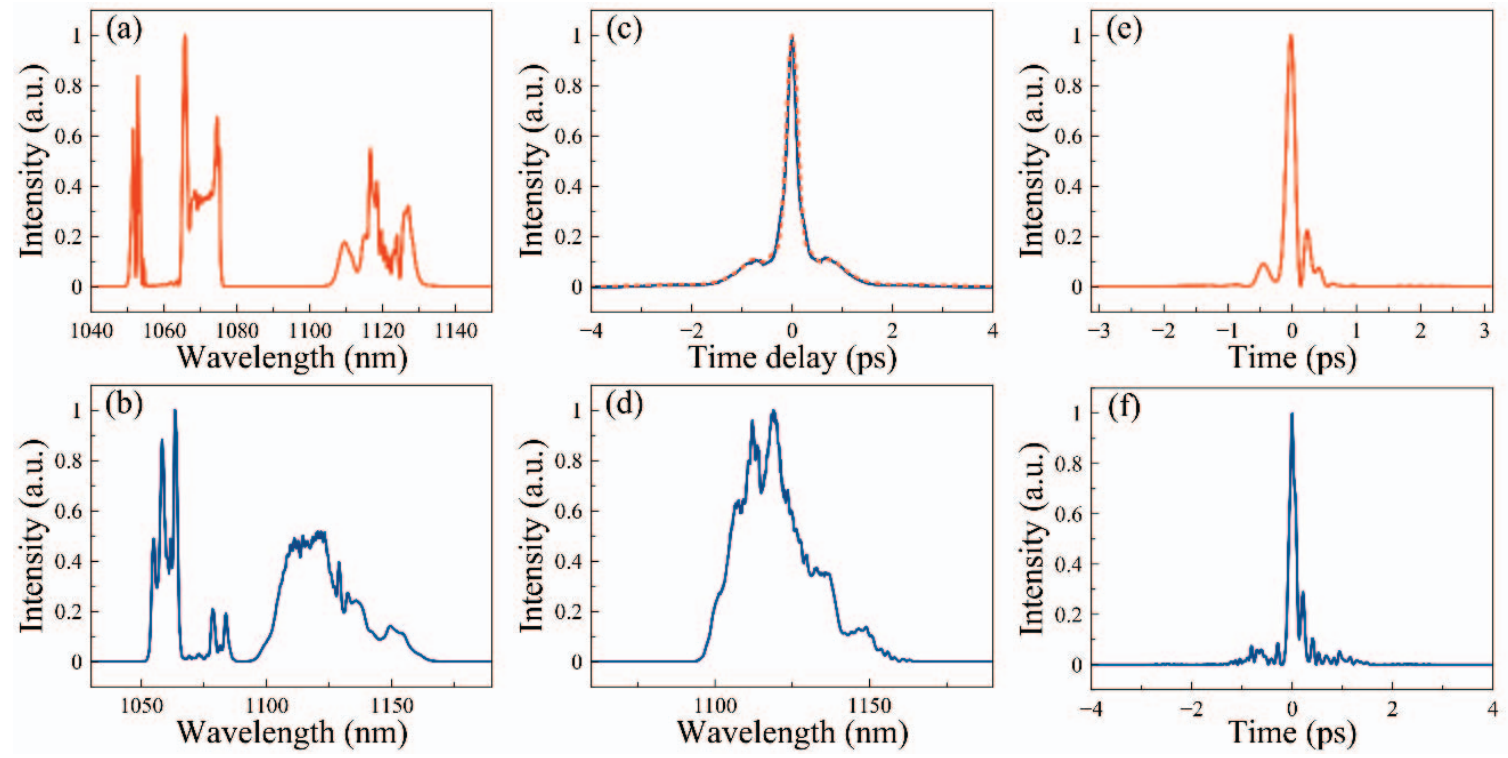

Fig. 2. (a) Numerical simulation and (b) measured spectra of Raman laser with internal feedback. (c) Measured autocorrelation trace of the dechirped pulse (blue solid-line) and calculated autocorrelation trace of dechirped pulse using PICASO algorithm (red dashed-line). (d)

Measured output Stokes spectrum after longpass filtering. (e) Numerical simulation and (f) retrieved pulse using PICASO algorithm

Stimulated Raman scattering was modeled using the analytical model presented in [6]. Gain was modeled by solving rate-equations using experimentally measured emission and absorption cross-sections of ytterbium, ignoring amplified spontaneous emission [7]. Figs 2(a) and 2(e) show the simulatied optical spectra and pulse shape, respectively.

PICASO algorithm [8] was used to infer the pulse shape from the measured autocorrelation and spectrum. The measured pulse width for the Raman pulse is 136 fs with Gaussian-shape time-bandwidth product. The PICASO-retrieved pulse shapes duration is $154 \mathrm{fs}$, as shown in Fig. $2 \mathrm{f}$.

In conclusion, we have demonstrated the first synchronously pumped dissipative soliton Raman laser. The pump laser comprises of an oscillator and an amplifier to generate highly-chirp pulses centered at $1060 \mathrm{~nm}$. By leading the pulses into Raman oscillator (the same length as pump oscillator), after generating Stokes pulses and re-launching it, we generated strong Raman pulses. The intra-cavity energy of Raman pulse is $7.1 \mathrm{~nJ}$ and after compressing, $136 \mathrm{fs}$ long pulses is achieved. The Raman oscillator can be modeled by complex Ginzburg-Landau equation, which support dissipative Raman solitons.

This work was supported by the Scientific and Technological Research Council of Turkey TÜBİTAK under grant no. $112 \mathrm{~T} 944$ and European Research Council (ERC) ERC-2013-CoG NLL.

\section{REFERENCES}

[1] R. H. Stolen, C. Lin, \& R. K. Jain, (1977). “A timedispersion-tuned fiber Raman oscillator". Applied Physics Letters, 30(7), 340.

[2] J. D. Kafka, \& T. Baer, (1987). "Fiber Raman soliton laser pumped by a Nd:YAG laser". Optics Letters, 12(3), 181.

[3] C. Aguergaray, D. Méchin, V. Kruglov, \& J. D. Harvey, (2010). "Experimental realization of a mode-locked parabolic Raman fiber oscillator". Optics Express, 18(2), 8680.

[4] P. Grelu, S. Chouli, J. M. Soto-Crespo, W. Chang, A Ankiewicz, \& N. Akhmediev, (2010). "Dissipative solitons for mode-locked fiber lasers". Nature Photonics, 6(February), 84.

[5] J. Hult, (2007). "A fourth-order Runge-Kutta in the interaction picture method for simulating supercontinuum generation in optical fibers". Journal of Lightwave Technology, 25(12), 3770.

[6] D. Hollenbeck \& C. D. Cantrell, (2002). "Multiplevibrational-mode model for fiber-optic Raman gain spectrum and response function". Journal of the Optical Society of America B, 19(12), 2886.

[7] R. Paschotta, J. Nilsson, A. C. Tropper, \& D. C. Hanna, (1997). "Ytterbium-doped fiber amplifiers". IEEE Journal of Quantum Electronics, 33(7), 1049.

[8] J. W. Nicholson, J. Jasapara, W. Rudolph, F. G. Omenetto \& J. Taylor, (1999). "Full-field characterization of femtosecond pulses by spectrum and cross-correlation measurements". Optics Letters, 24(23), 1774. 